\title{
The 8th Japan Bioanalysis Forum symposium
}

\author{
Takehisa Matsumaru*,1 \\ ${ }^{1}$ Otsuka Pharmaceutical Co., Ltd., 3-2-27 Otedori, Chuo-ku, Osaka 540-0021, Japan \\ * Author for correspondence: Tel.: +81 66943 7722; matsumaru.takehisa@otsuka.jp
}

The 8th Japan Bioanalysis Forum symposium, the Tower Hall Funabori, Tokyo, Japan, 8-9 February 2017

The 8th Japan Bioanalysis Forum (JBF) symposium was successfully held between 8 and 9 February 2017 at the Tower Hall Funabori, Tokyo, Japan. In total, 24 speakers from Japan, USA and Europe gave presentations regarding the immunogenicity of biopharmaceuticals, ICH S3A Q\&A microsampling, ICH M10 bioanalytical method validation, large molecule analysis through LC-MS, auditing activities for bioanalysis and biomarker bioanalysis. Achievements regarding eight diverse themes were also shared by Japan Bioanalysis Forum discussion groups. Over 300 scientists from regulatory agencies, industry and academia actively took part in discussions during the symposium. This article provides the highlights of all the topics discussed in this symposium.

First draft submitted: 1 September 2017; Accepted for publication: 20 September 2017; Published online: 9 November 2017

Keywords: ADA $\bullet$ bioanalysis $\bullet$ bioanalytical method validation $\bullet$ biomarker $\bullet$ discussion group $\bullet$ JBF $\bullet$ LC-MS

\section{Immunogenicity testing of biopharmaceuticals}

Akiko Ishii-Watabe (National Institute of Health Sciences [NIHS]) presented the current status of immunogenicity assessments in Japan. Many biotherapeutics have been approved in Japan with immunogenicity assessments performed based either on a basic concept widely accepted amongst bioanalysts in the US or a similar one shared in the EU due to there being no specific guideline yet in Japan. The US FDA guidance and the draft EMA guideline currently serve as good references for immunogenicity assessment in Japan [1,2]. To ensure the reliability of anti-drug antibody (ADA) assays, important considerations are currently under discussion by the Japan Agency for Medical Research and Development (AMED) immunogenicity research group. The topics include cut-point determination, drug tolerance, biosimilars and reporting.

William Hallett (FDA) gave an overview of the FDA draft guidance for immunogenicity testing updated in 2016 [1], especially focusing on immunoglobulin isotypes, assay sensitivity and general considerations for assay validation. He recommended that screening assays should be able to detect all relevant immunoglobulin isotypes and that ADA assays need to be sufficiently sensitive and ready to detect these at a sufficiently low level so that the pharmacokinetics (PK), PD, safety or efficacy profiles are not affected.

Meenu Wadhwa (National Institute for Biological Standards and Control, UK) covered the European guidance for immunogenicity therapeutic proteins. The European guidance consists of several guidelines [2-4] and the Guideline on Immunogenicity Assessment of Biotechnology-Derived Therapeutic Proteins published in 2008 [2] is currently being revised [5]. The new guideline will be aligned with other related guidelines and will include a risk-based approach and specific information on ADA assays. It will also cover immunogenicity testing for biosimilars and the issues of pharmacovigilance.

Jo Goodman (European Bioanalysis Forum [EBF]) introduced recent EBF activities related to immunogenicity and the feedback of the Focus Workshop on 'Current analysis of immunogenicity - Best Practices and Regulatory Hurdles' held in Lisbon in 2016 [6]. The EBF summarized the comments on both the EMA and FDA draft documents and the outcome of the industry survey conducted to keep a finger on the pulse of immunogenicity data submissions. The Focus Workshop covered broad topics on immunogenicity testing, including the regulatory landscape, drug tolerance, target interference, alternatives for neutralizing antibody assessment and cut-point setting. 


\section{ICH S3A Q\&A microsampling \& ICH M10 bioanalytical method validation}

First, Yoshiro Saito (NIHS) presented the current status of ICH S3A Q\&A (Questions and Answers: Note for Guidance on Toxicokinetics: The Assessment of Systemic Exposure - Focus on Microsampling) and ICH M10 Bioanalytical Method Validation. Each country completed public consultation on draft ICH S3A Q\&A and is currently working on public comments. Additionally, ICH is busy with preparing the first draft of the ICH M10 guideline and plans to reach step two in 2018.

Yoshiaki Ohtsu (Japan Bioanalysis Forum [JBF]; Astellas Pharma) shared the expectation for ICH M10 from JBF's viewpoint and mentioned that the requirement for internal standards (ISs) can be optimized by considering the role of ISs in analytical procedures.

Philip Timmerman (Janssen R\&D), representing EBF, gave feedback from discussions at the 9th EBF Open Symposium on the industry's opinion of what a modern bioanalytical guideline should consider. It was highlighted that the principles of scientific validation should be recognized by a modern guideline. The audience also felt that a modern guideline should consider the industry's experience in areas of ambiguity or where experiments have shown that there is no added value to generating data.

Finally, Faye Vazvaei (Roche) discussed American Association of Pharmaceutical Scientists (AAPS) activities in regulated bioanalysis and pointed out some noteworthy philosophical versus procedural differences among US/EU/Japan regulatory documents and provided AAPS' initial thoughts on areas with higher priority for harmonization. Importantly, JBF, EBF and AAPS all agreed that the bioanalysis of biomarkers and ADA should be outside the scope of a PK assay guideline.

\section{Advancement of large-molecule analysis through LC-MS}

Recently, LC-MS has become a practical approach for protein quantifications as an alternative to ligand-binding assays.

Noritaka Hashii (NIHS) discussed the bioanalytical challenges he encountered regarding trastuzumab in human serum through LC-MS. He successfully achieved the LLOQ at $50 \mathrm{ng} / \mathrm{ml}$ by using a stable isotope labeled Fab.

Takashi Shimada (Shimadzu) presented nano-surface and molecular-orientation limited (nSMOL) proteolysis [7]. The FC region of the IgG fraction was immobilized on the inner surface of the resin pore $(100 \mathrm{~nm})$ and only the Fab region protruded from the pore. Additionally, proteolysis was performed using immobilized trypsin on the surface of nanoparticles $(200 \mathrm{~nm})$. Owing to diameter difference between the resin pore and the nanoparticle, limited proteolysis on antibody Fab was enabled with minimized complexity or protease contamination. Validation studies of nSMOL coupled with LC-MS have already been achieved with several therapeutic antibodies and multiplex assays in the human plasma [8,9]. The LLOQ for trastuzumab was $50 \mathrm{ng} / \mathrm{ml}$. Clinical trials using $\mathrm{nSMOL}$ assay are now in progress in several medical centers.

Sumio Ohtsuki (Kumamoto University) gave a comprehensive presentation on the advancement and divergence of LC-MS-based protein quantitation. Originally, multiple reaction monitoring using a triple-quadrupole mass spectrometer has been used for protein quantification. Recently, due to the improvement in performance, high resolution mass spectrometers (HR-MS), such as the Q-TOF type, have also been used for quantification. In addition, Data Independent Acquisition (DIA, SWATH-MS) has been developed and its application in quantification has been reported [10]. It is important to understand the advantages and disadvantages of each method for each application. The results indicated that SWATH-MS enabled large-scale multiplex absolute protein quantification while retaining similar quantitative capability to multiple reaction monitoring or HR-MS.

\section{JBF DG activities}

Yoshihisa Sano (JBF; Sunplanet) organized this session. The leaders of eight Discussion Groups (DGs; DG20-27) introduced their activities briefly in the oral session on Day 1 and the members of each DG presented their outcomes and openly exchanged their views on a wide range of bioanalytical issues with symposium attendees in the poster session, which took place on Day 2.

DG20 succeeded working on the topic 'scientific validation', which was previously discussed in DG16 (2015). The group consisted of 12 members (leader: Makoto Niwa; Nippon Kayaku). They presented the outcome of the survey to Japanese scientists regarding the acceptance levels of the scientific validation items proposed by EBF [11]. The applicability of the scientific validation items to in vitro studies was also surveyed and shared with the attendees.

DG21 focused on the topic 'Microsampling (2): Investigation of Technical Problems'. The group consisted of 11 members (leader: Katsunori Ieki; Shin Nippon Biomedical Laboratories). They discussed the following issues: 
'Merits and Demerits of Microsampling Methods and Devices', 'Effects on PK Data by Differences in Blood Sampling Sites and Physiological Effects of Blood Sampling', 'Improvement of Analytical Method Sensitivity', 'Additional Validation when Analyzing Samples Derived from Microsampling,' and 'Factors Affecting Accuracy and Precision in Quantitative Analysis' and proposed countermeasures to the technical problems of microsampling.

DG22 discussed the topic 'Application of Imaging Mass Spectrometry (IMS) to Drug Discovery Research'. The group consisted of four members (leader: Yukari Tanaka; Shionogi). They engaged in many exciting discussions regarding the applications of IMS and current technological directions in drug discovery research. In terms of the technological issues of IMS, they discussed ion suppression in matrix-assisted laser desorption/ionization (MALDI)-IMS analysis, how to normalize ion intensities in MALDI-IMS analysis and how to apply MALDI matrices to tissue sections. The application of IMS to toxicity evaluation was also discussed.

DG23 discussed a topic entitled 'Pharma/CRO Relationship with a Focus on Method Transfer/Development'. The group consisted of four members from pharmaceutical companies and two members from CROs (leader: Yoshitaka Hashimoto; Ono Pharmaceutical). The group surveyed a wide range of items regarding pharma/CRO co-work for method development/transfer (e.g., current challenges, outsourcing strategy, strengths and weaknesses of each side, differences between Japanese and foreign CROs, how to define and share necessary information, how to communicate with a counterpart and the case study of a failure) and shared the findings and their thoughts from the standpoints of both a pharmaceutical company and CRO.

DG24 discussed the topic 'Questions and Challenges in Bioanalysis: Find the Loadstar (Focusing on Stability)' in small-molecule quantification. The group consisted of eight members (leader: Yuya Hosokawa; Ono Pharmaceutical). They first introduced the stability recommendation in a Global Bioanalysis Consortium (GBC) publication [12]. They then surveyed DG supporters and after receiving many questions on stability in matrices, stability related to metabolites and stability related to special matrices (blood or tissue); they then proposed some solutions and recommendations.

DG25 consisted of seven members (leader: Akira Wakamatsu; GlaxoSmithKline), who discussed the topic 'Quantitative Analysis of Endogenous Substances (by LC-MS)' following the last two years' discussions in DG08 (2014) and DG15 (2015). This time, they focused on two topics, 'The Quantitative Analysis of Large-Molecule Endogenous Substances (LM) by LC-MS' and 'The Quantitative Analysis of Endogenous Substances with Surrogate Analytes (SA)'. For the analysis of large-molecule endogenous substances, they confirmed the definition of 'absolute/relative quantification' and delved into cleanup, enzyme digestion and IS spiking. For the use of SA, they discussed points to consider in the selection of SA. Additionally, they proposed a strategy for using SA and surrogate matrix.

DG26 discussed the topic 'Quantitative Analysis of Endogenous Substances (by LBA)' following the consecutive discussion from DG15 (2015). The group consisted of seven members (leader: Satomi Sasahara; Towa Pharmaceutical). They discussed the implementation and evaluation of method validation for the quantitative analysis of endogenous substances using ligand-binding assay (LBA). They dealt with two sorts of analytes (recombinant proteins and endogenous substances) separately in their discussion because structures of endogenous substances and standard substances (i.e., recombinant proteins) are not the same.

DG27 focused on the quantitative analysis of endogenous substances with flow cytometry, Luminex and PCR. The group consisted of eight members (leader: Takahiro Nakamura; Shin Nippon Biomedical Laboratories). Regarding flow cytometry, they mainly discussed the method development and validation of immunophenotyping. They also discussed bead arrays and flow cytometry-based target receptor occupancy. Furthermore, they discussed method development and validation by Luminex in the quantitative analysis of endogenous substances. Moreover, method development and validation by quantitative real-time PCR were discussed in a dose-determining assay of transgene or cells with a focus on the specific characteristics of PCR.

\section{Discussion regarding audit activities for bioanalysis study}

The Japan Society of Quality Assurance (JSQA) considers the quality assurance of medicines and technical proposals and Joint Special Project Group 2 examines quality assurance methodology at clinical/medical laboratories for clinical trials. They conducted a questionnaire survey on the 'audit of bioanalysis studies' targeted towards JBF partners. Tomoyoshi Taniguchi (Eisai) and Kayo Takahashi (the Research Foundation for Microbial Diseases of Osaka University) presented the survey results on the audit of bioanalysis in the oral and poster sessions, respectively. Yoshinobu Yamai (Taisho Pharmaceutical) suggested the audit activities based on their discussion regarding several gaps between analysts and auditors for the bioanalysis study in the oral session. Mayumi Hayashi 
(JCR Pharmaceuticals) presented the main points of their discussion regarding the 'auditor's perspective for bioanalysis in clinical trials' in the poster session.

\section{Biomarker bioanalysis \& its application to drug development}

Though the concept of the fit-for-purpose approach in biomarker bioanalysis is widely accepted among global bioanalysts, there is still much discussion of how to apply this concept to each bioanalytical method during drug development. In this session, three speakers presented their experiences of the practical implementation of the bioanalysis of biomarkers in several phases of drug development.

Shinya Horita (Kyowa Hakko Kirin) presented the outcomes from his biomarker bioanalysis in an early-stage clinical trial. Multiple biomarkers, including endogenous ligands, cytokines and cytokine receptor-expressed cells, were analyzed in the study through LC-MS/MS, ligand binding assays and flow cytometry, respectively. Each bioanalytical method was validated at an adequate level to satisfy the purpose of the analysis.

The second presentation was given by Makoto Yamazaki (Mitsubishi Tanabe Pharma) on the bioanalysis of biomarkers in preclinical phases. He introduced three cases of biomarker bioanalysis in different stages of the preclinical phase (discovery, early preclinical and late preclinical). The requirements for preclinical biomarker bioanalysis validations differ depending on the stages and characteristics of analytes and this point was discussed in this presentation.

Finally, Kazuaki Sakai (Teijin Pharma) presented an interesting perspective on the quantitative analysis of endogenous substances based on the discussion in DG2015-15. Both the selection process of an adequate surrogate matrix and its importance were explained.

\section{Conclusion}

Significant progress in regulatory bioanalysis was reported regarding immunogenicity assessments and ICH topics by speakers from regulatory agencies and industries. Actual works on large-molecule analysis through LC-MS or biomarker assays provided very helpful information for bioanalytical scientists who are exploring these fields.

The data integrity in bioanalytical results is a critical factor for ensuring the quality of medical drugs. We have to contribute to delivering safe and effective pharmaceuticals swiftly to patients by facilitating scientific discussions on bioanalysis among scientists, bioanalytical communities and regulatory agencies.

Most presentation materials are available on the JBF website [13]. JBF has continuously served as the sole forum for regulated bioanalysis in Japan and the 9th JBF symposium will be held on 6-8 February 2018 in Tokyo, Japan.

\section{Acknowledgements}

As the symposium organizer, the author would like to thank: Shinichi Miura (JPMA/Daiichi Sankyo) and Japan Society of Quality Assurance for their fruitful sessions, all speakers for their meaningful presentations and opinions, JBF DG members and symposium attendees for their attentive discussion and the JBF steering committees for their dedication.

\section{Financial \& competing interests disclosure}

The author has no relevant affiliations or financial involvement with any organization or entity with a financial interest in or financial conflict with the subject matter or materials discussed in the manuscript. This includes employment, consultancies, honoraria, stock ownership or options, expert testimony, grants or patents received or pending, or royalties.

No writing assistance was utilized in the production of this manuscript.

\section{References}

1. Draft Guidance for Industry: Assay development and validation for immunogenicity Testing of Therapeutic Protein Products. US Department of Health and Human Services, US FDA, Rockville, MD, USA (2016). www.fda.gov/downloads/Drugs/Guidances/UCM192750.pdf

2. Guideline on Immunogenicity assessment of biotechnology-derived therapeutic proteins. European Medicines Agency, London, UK (2008). www.ema.europa.eu/docs/en_GB/document_library/Scientific_guideline/2009/09/WC500003946.pdf

3. Guideline on immunogenicity assessment of monoclonal antibodies intended for in vivo clinical use. European Medicines Agency, London, UK (2012). www.ema.europa.eu/docs/en_GB/document_library/Scientific_guideline/2012/06/WC500128688.pdf

4. Guideline on similar biological medicinal products containing monoclonal antibodies - non-clinical and clinical issues. European Medicines Agency, London, UK (2012). www.ema.europa.eu/docs/en_GB/document_library/Scientific_guideline/2012/06/WC500128686.pdf 
5. Draft Guideline on Immunogenicity assessment of biotechnology-derived therapeutic proteins. European Medicines Agency, London, UK (2015). www.ema.europa.eu/docs/en_GB/document_library/Scientific_guideline/2015/10/WC500194507.pdf

6. EBF - Focus Workshop: Current analysis of immunogenicity - Best Practices and Regulatory Hurdles. http://focus201609.europeanbioanalysisforum.eu/slides-2/

7. Iwamoto N, Shimada T, Umino Y et al. Selective detection of complementarity-determining regions of monoclonal antibody by limiting protease access to the substrate: nano-surface and molecular-orientation limited proteolysis. Analyst 139(3), 576-580 (2014).

8. Iwamoto N, Yamane N, Umino Y et al. The developmentof the validated LCMS bioanalysis of trastuzumab in human plasma using aselective detection method for complementarity-determining regions of monoclonal antibodies: nano-surface and molecular-orientation limited (nSMOL) proteolysis. Anal. Methods 7, 9177-9183 (2015).

9. Iwamoto N, Takahashi M, Hamada A et al. Multiplex LCMS bioanalysis of brentuximab vedotin rituximab and cetuximab towards therapeutic drug monitoring application by combined calibration curve using fab-Selectivelimited proteolysis nSMOL. Clin. Pharmacol. Biopharm. 5, 164 (2016).

10. Nakamura K, Hirayama-Kurogi M, Ito $S$ et al. Large-scale multiplex absolute protein quantification of drug-metabolizing enzymes and transporters in human intestine, liver, and kidney microsomes by SWATH-MS: Comparison with MRM/SRM and HR-MRM/PRM. Proteomics 16(15-16), 2106-2117 (2016).

11. Timmerman P, White S, McDougall S et al. Tiered approach into practice: scientific validation for chromatography-based assays in early development - a recommendation from the European Bioanalysis Forum. Bioanalysis 7(18), 2387-2398 (2015).

12. van de Merbel N, Savoie N, Yadav M et al. Stability: Recommendation for best practices and harmonization from the Global Bioanalysis Consortium Harmonization Team. AAPS J. 16(3), 392-399 (2014).

13. Japan Bioanalysis Forum 8th JBF Symposium. http://bioanalysisforum.jp/en/8th_JBFsympo_info 
